\title{
Association between low expression levels of interleukin-9 and colon cancer progression
}

\author{
YONGHONG HUANG $^{1}$, YUNFEI CAO ${ }^{2}$, SEN ZHANG ${ }^{2}$ and FENG GAO ${ }^{2}$ \\ ${ }^{1}$ Department of Gastroenterological Surgery, Cancer Center of Guangzhou Medical University, \\ Guangzhou, Guangdong 510095; ${ }^{2}$ Department of Colorectal and Anal Surgery, \\ First Affiliated Hospital of Guangxi Medical University, Nanning, Guangxi 530021, P.R. China
}

Received August 7, 2014; Accepted April 30, 2015

DOI: $10.3892 / \mathrm{etm} .2015 .2588$

\begin{abstract}
Although interleukin (IL)-9 has been extensively studied in inflammation and autoimmune diseases, the expression level of IL-9 in colon cancer and its clinical significance are less well established. In total, 15 healthy donors (HDs) and 60 patients who had been diagnosed with colon cancer that had undergone a surgical resection were enrolled in the study. The plasma levels of IL-9 in the HDs and cancer patients were detected by the liquid chip technique, while the expression levels of IL-9 in the colon cancer tissues and normal tissues were analyzed using immunohistochemistry (IHC) and reverse transcription-quantitative polymerase chain reaction (RT-qPCR). Finally, the difference in the expression levels of IL-9 between the patients classified as tumor-node-metastasis stage I-II and stage III-IV was compared. The results demonstrated that the plasma levels of IL-9 in the patients with colon cancer were significantly lower when compared with the HDs (1.29 vs. $2.53 \mathrm{pg} / \mathrm{ml}, \mathrm{P}<0.05)$. Furthermore, according to the IHC and RT-qPCR results, low expression levels of IL-9 were observed in the colon cancer tissues when compared with the normal tissues $(\mathrm{P}<0.05)$. With regard to the plasma and tumor tissue samples, patients diagnosed with stage III-IV colon cancer expressed lower levels of IL-9 compared with the stage I-II patients $(\mathrm{P}<0.05)$. In conclusion, low expression levels of IL-9 were observed in the tissue and plasma samples collected from the colon cancer patients, and the decreased expression of IL-9 was shown to correlate with colon cancer progression.
\end{abstract}

Correspondence to: Professor Yunfei Cao or Professor Feng Gao, Department of Colorectal and Anal Surgery, First Affiliated Hospital of Guangxi Medical University, 6 Shuangyong Road, Nanning, Guangxi 530021, P.R. China

E-mail: caoyunfei126@126.com

E-mail: doctorgao0771@hotmail.com

Key words: interleukin-9, colon cancer, tumor-node-metastasis stage

\section{Introduction}

Colon cancer is one of the most commonly diagnosed cancers among men and women (1). Since the introduction and dissemination of early detection tests, in addition to improvements in treatment, an increased overall survival rate has been observed in patients with colon cancer; however, the disease remains the third leading cause of cancer-associated mortality in both men and women (2). Research into tumor immunology has developed rapidly in recent years, which has aided improvements in the prognosis of malignance (3).

Interleukin (IL)-9 was first described in the late 1980s as a member of a growing number of cytokines that exhibited pleiotropic functions in the immune system (4). IL-9 is a member of the common $\gamma$ chain cytokine family, with other members including IL-2, IL-4, IL-7, IL-15 and IL-21 (5). IL-9 exerts its function by binding to the IL-9 receptor (IL-9R), a heterodimer complex consisting of the IL-9R $\alpha$ and the common $\gamma$ chain (6). The cellular sources of IL-9 are diverse, and include multiple $\mathrm{T}$ helper cell subsets, regulatory $\mathrm{T}$ cells, mast cells and natural killer T cells (5). IL-9 has been extensively studied in lung inflammation (7-10), parasitic infection $(11,12)$ and autoimmune disease (13-16); however, the function of IL-9 in tumor immunity remains unclear and controversial $(17,18)$. In the current study, the expression level of IL-9 was investigated in the peripheral blood and tumor tissues of patients with colon cancer, and the association between the expression level of IL-9 with tumor stage was assessed.

\section{Materials and methods}

Ethics statements. All patients and healthy donors (HDs) enrolled in the study provided written informed consent. The study protocol conformed to the ethical guidelines of the Declaration of Helsinki (Fortaleza, Brazil, October 2013), and was approved by the Ethical Committees and Institutional Review Board of the First Affiliated Hospital of Guangxi Medical University (Nanning, China).

Research subjects and samples. Between June 2013 and June 2014, 60 patients who had been diagnosed with colon cancer and had undergone surgical treatment in the First Affiliated Hospital of Guangxi Medical University were 
enrolled in the study. None of patients had received radiotherapy or chemotherapy prior to sampling. Individuals with an autoimmune disease, an infectious disease or multiple primary cancers were excluded. An additional 15 HDs were enrolled in the study. Fresh peripheral blood samples were obtained from the 60 patients and 15 HDs in the morning. In addition, fresh tumor tissues and normal colon tissues (at least $5 \mathrm{~cm}$ distant from the tumor site) were obtained from the patients who underwent colorectal cancer resection surgery. The tissue specimens were collected with liquid nitrogen for polymerase chain reaction (PCR) analysis or fixed with $4 \%$ paraformaldehyde for immunohistochemistry (IHC).

$I H C$. Fresh tumor and normal colon tissues were fixed in $4 \%$ paraformaldehyde, embedded with paraffin and sectioned at $4-\mu \mathrm{m}$ thickness. IHC was performed as previously described (19). The sectioned slides were stained immunohistochemically for IL-9, which was purchased from Beijing Biosynthesis Co., Ltd. (Beijing, China).

Liquid chip technique. Peripheral blood samples were collected in heparinized tubes (Shenzhen Medrey Medical Technology Co., Ltd., Shenzen, China). Subsequently, the plasma was obtained by centrifugation at $1,048 \mathrm{x}$ g for $20 \mathrm{~min}$, and stored at $-80^{\circ} \mathrm{C}$ until required for analysis. Luminex analysis was performed using a ProcartaPlex ${ }^{\mathrm{TM}}$ Multiplex Immunoassay (eBioscience, Vienna, Austria) to determine the plasma level of IL-9, according to the manufacturer's instructions.

Reverse transcription-quantitative PCR (RT-qPCR). Fresh tumor and normal colon tissue samples, collected for the determination of IL-9 mRNA expression levels, were stored at $-80^{\circ} \mathrm{C}$ until required for analysis. Total RNA was extracted using TRIzol reagent (Invitrogen Life Technologies, Carlsbad, CA, USA), according to the manufacturer's instructions. The extracted RNA was immediately reverse transcribed to form cDNA using a SuperScript III First-Strand Synthesis System (Invitrogen Life Technologies). Subsequently, RT-qPCR was performed using the SYBR Green PCR kit (Roche Diagnostics, Basel, Switzerland). Amplification was performed under standard conditions (20) and the expression levels of IL-9 were normalized against the transcripts of the housekeeping gene, $\beta$-actin. The following primers were used for RT-qPCR: IL-9 forward, 5'-CTC TGT TTG GGC ATT CCC TCT-3' and reverse, 5'-GGG TAT CTT GTT TGC ATG GTG G-3'; $\beta$-actin forward, 5'-TGA CGT GGA CAT CCG CAA AG-3' and reverse, 5'-CTG GAA GGT GGA CAG CGA GG-3'. Relative expression levels of mRNA were calculated using the $2^{-\Delta \Delta \mathrm{Ct}}$ method, as described by Livak and Schmittgen (21), and adjusted according to the level of $\beta$-actin mRNA for each sample.

Statistical analysis. Data are expressed as the mean \pm standard error. Comparisons of the data between different groups were conducted using the Student's t-test, the paired t-test or one-way analysis of variance. Statistical analysis was completed using GraphPad Prism 5.0 software (GraphPad Software, Inc., San Diego, CA, USA), and $\mathrm{P}<0.05$ was considered to indicate a statistically significant difference.

\section{Results}

Low plasma levels of IL-9 in colon cancer patients. Plasma levels of IL-9 were determined by Luminex analysis. In four patients, the concentration of IL-9 was too low to be detected. Thus, the mean concentration of plasma IL-9 for 56 patients was $1.29 \mathrm{pg} / \mathrm{ml}$ (range, 0.11-4.31 pg/ml). Plasma IL-9 levels were detected in all the $15 \mathrm{HDs}$, and the average concentration was $2.53 \mathrm{pg} / \mathrm{ml}$ (range, 0.06-9.84 pg/ml), which was significantly higher when compared with the patients with colon cancer $(\mathrm{P}<0.05$; Fig. 1A).

Low expression levels of IL-9 in colon cancer tissues. With regard to the IHC results, IL-9 expression was observed in the colon cancer tissues and the normal colon tissues, although expression in the normal tissues appeared more evident (Fig. 2). In accordance, RT-qPCR was performed to investigate the mRNA expression levels of IL-9, which were adjusted according to the level of $\beta$-actin. As shown in Fig. 1B, the relative mRNA expression levels of IL-9 in the colon cancer tissues were significantly lower when compared with that in the normal tissues $(\mathrm{P}<0.05)$.

Low expression levels of IL-9 are associated with tumor progression. The clinical significance of IL-9 expression levels in the plasma and colon tissues was analyzed. According to the tumor-node-metastasis (TNM) staging system, the plasma level of IL-9 in the patients classified as stage III-IV was significantly lower compared with that in the patients classified as stage I-II ( $\mathrm{P}<0.05$; Fig. 3A). In addition, similar results were observed in the relative mRNA expression levels of IL-9 in the colon cancer tissues $(\mathrm{P}<0.05$; Fig. $3 \mathrm{~B})$.

\section{Discussion}

IL-9 is a member of the common $\gamma$ chain cytokine family, which participates in a variety of inflammatory and autoimmune diseases (22). IL-9 promotes allergic responses in the lungs by stimulating mast cell expansion and the production of IL-13, which in turn induces the release of mucus that contributes to airway hyper-responsiveness $(7,8)$. Therefore, targeting the IL-9 pathway may provide a novel therapeutic modality for asthma $(9,10)$.

In addition to having a pathogenic role in the allergic response in the lungs, IL-9 is also associated with the development of autoimmune diseases (13-15). Using an animal model, an IL-9 blockade with anti-IL-9 monoclonal antibodies was shown to inhibit the development of experimental autoimmune encephalomyelitis (16). However, IL-9 has been shown to provide a protective role in immunity to intestinal parasites by promoting the clearance of worms $(11,12)$. Furthermore, rather than T helper 9 (Th9) cells, regulatory T cells appear to be one of the major sources of IL-9, which have been hypothesized to be involved in the recruitment of mast cells that mediate the tolerance of the skin (23) and liver transplantation (24).

The roles of IL-9 in tumor immunity remain unclear and controversial. A large number of studies have confirmed that overexpression of IL-9 is associated with the progression of various forms of lymphoma and 
A

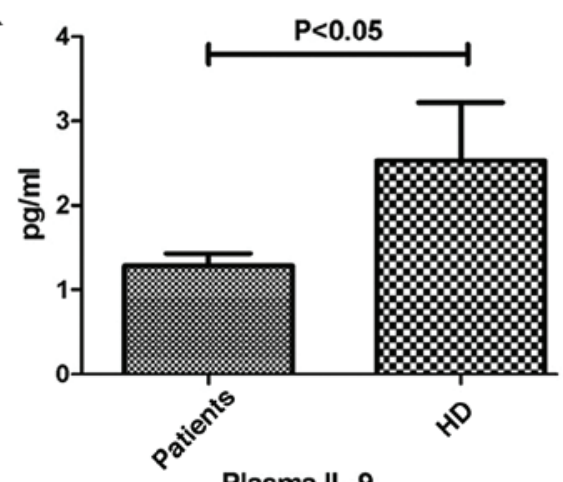

B

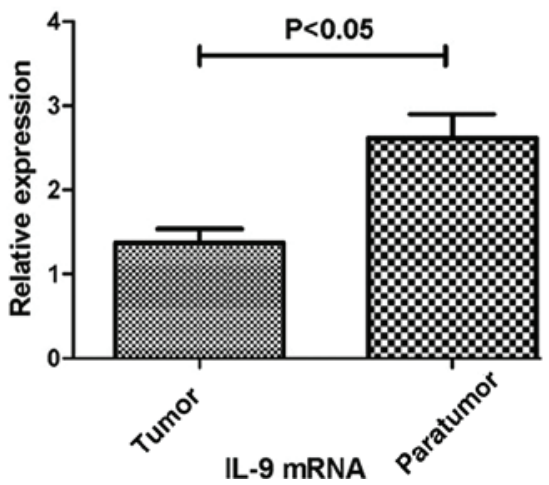

Figure 1. Expression levels of IL-9 in the plasma and tissue samples. (A) Plasma levels of IL-9 in the colon cancer patients and HDs. (B) Expression levels of IL-9 in the colon cancer tissues (tumor) and normal colon tissues (normal) were determined by reverse transcription-quantitative polymerase chain reaction. Relative expression was normalized against the level of $\beta$-actin mRNA for each sample. Each bar represents the mean \pm standard error. IL, interleukin; HD, healthy donors.
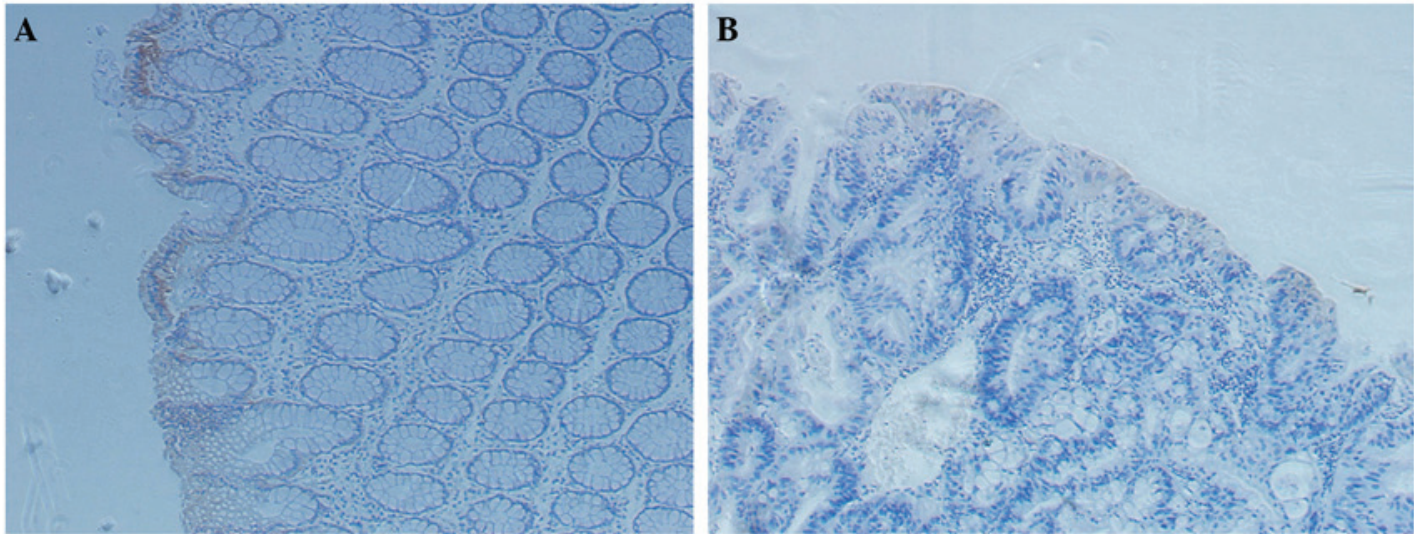

Figure 2. Immunohistochemistry (IHC) staining of the tissues (Magnification, x200). IHC staining for (A) paratumor tissues and (B) colon cancer tissues.

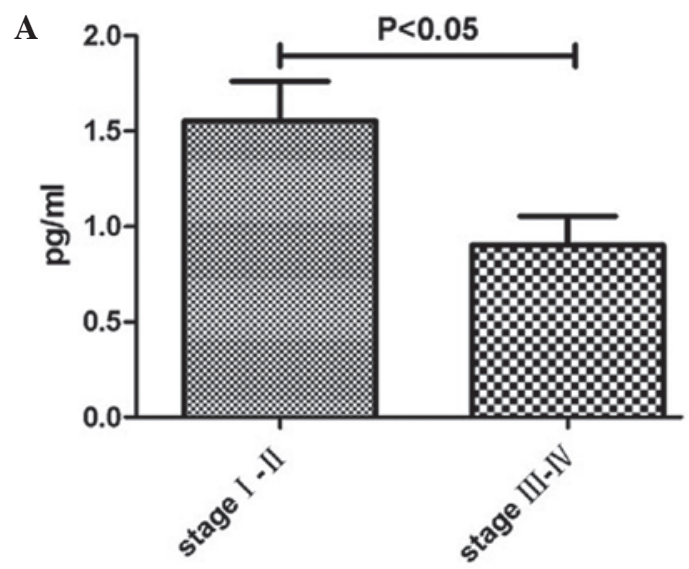

Plasma IL-9

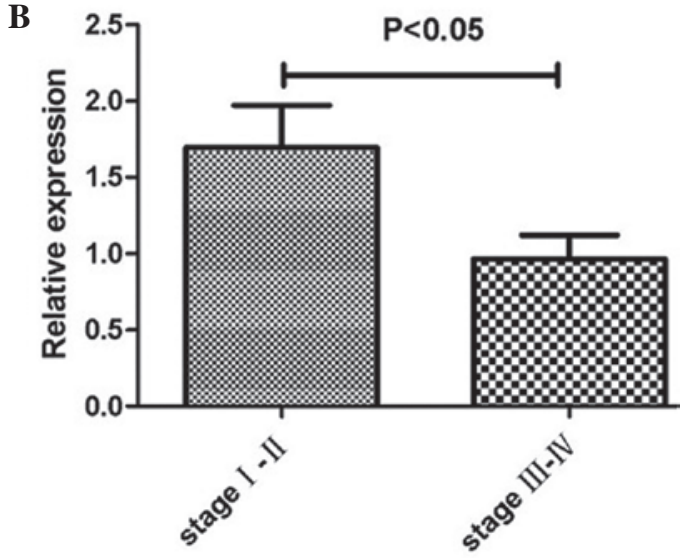

IL-9 mRNA

Figure 3. Differences in the expression levels of IL-9 between patients classified as tumor-node-metastasis (TNM) stage I-II and stage III-IV. Differences in the expression levels of IL-9 in the (A) plasma and (B) tumor tissues between patients classified as TNM stage I-II and stage III-IV. Each bar represents the mean \pm standard error. IL, interleukin.

leukemia (25-29), possibly through the activation of the Janus kinase/signal transducer and activator of transcription (STAT) pathway to promote cancer development (30-33). In addition, the blockade of IL-9 may serve as a novel strategy to modulate the function of regulatory $\mathrm{T}$ cells to subsequently enhance the antitumor effect of tumor vaccines (18). Recruitment of IL-9 and Th9 cells has also been demonstrated in malignant pleural effusion by activating STAT3 signaling, where IL-9 has been shown to substantially promote the proliferation and migratory activity of lung cancer cells (34). Nevertheless, two 
previous studies have reported that IL-9-producing $\mathrm{T}$ cells were associated with potent anticancer effects and favorable outcomes of cancer-bearing mice in an aggressive B16 melanoma model, which may be due to the effects of IL-9 on mast cells (17) or through eliciting a host antitumor $\mathrm{CD} 8^{+}$cytotoxic T lymphocyte response (35).

In the present study, the average concentration of the plasma IL-9 level was $1.29 \mathrm{pg} / \mathrm{ml}$ in the patients with colon cancer, which was significantly lower compared with the concentration of $2.53 \mathrm{pg} / \mathrm{ml}$ in the HDs $(\mathrm{P}<0.05)$. This observation was contrary to a previous study investigating lymphoma (24), but similar to a study in patients with skin melanoma (17). In addition, the present study demonstrated a low expression level of IL-9 in the colon cancer tissues when compared with the paratumor tissues, according to the IHC and PCR results. Reduced levels of IL-9 have also been demonstrated in an ovarian cancer ascites environment (36). Collectively, these results indicate that the expression level of IL-9 varies between cancer types. The generation of IL-9 may be inhibited in a colon cancer microenvironment, but activated in a lymphoma or leukemia microenvironment.

The TNM classification of malignant tumors is widely used in clinical practice. In the present study, patients classified with stage III-IV colon cancer were demonstrated to express lower levels of IL-9 when compared with the stage I-II patients, both in the plasma and tumor tissue samples, indicating that IL-9 may be used as a marker to predict the prognosis of patients with colon cancer.

In conclusion, to the best of our knowledge, the present study is the first to describe the expression level of IL-9 in patients with colon cancer, and confirm the close association between IL-9 and the TNM stage. Low expression levels of IL-9 were observed in the tissue and plasma samples collected from the colon cancer patients. Furthermore, decreased expression levels of IL-9 were shown to correlate with colon cancer progression. Therefore, these observations indicate that low expression of IL-9 may be associated with colon cancer development and progression.

\section{Acknowledgements}

This study was supported by grants from the National Natural Science Foundation of China (nos.81260316 and 81260335).

\section{References}

1. Jemal A, Bray F, Center MM, et al: Global cancer statistics. CA Cancer J Clin 61: 69-90, 2011.

2. Siegel R, Desantis C and Jemal A: Colorectal cancer statistics, 2014. CA Cancer J Clin 64: 104-117, 2014.

3. Bindea G, Mlecnik B, Angell HK and Galon J: The immune landscape of human tumors: Implications for cancer immunotherapy. OncoImmunology 3: e27456, 2014.

4. Goswami R and Kaplan MH: A brief history of IL-9. J Immunol 186: 3283-3288, 2011.

5. Noelle RJ and Nowak EC: Cellular sources and immune functions of interleukin-9. Nat Rev Immunol 10: 683-687, 2010.

6. Demoulin JB and Renauld JC: Interleukin 9 and its receptor: an overview of structure and function. Int Rev Immunol 16: 345-364, 1998.

7. Kearley J, Erjefalt JS, Andersson C, et al: IL-9 governs allergen-induced mast cell numbers in the lung and chronic remodeling of the airways. Am J Respir Crit Care Med 183: $865-875,2011$.
8. Steenwinckel V, Louahed J, Orabona C, et al: IL-13 mediates in vivo IL-9 activities on lung epithelial cells but not on hematopoietic cells. J Immunol 178: 3244-3251, 2007.

9. Oh CK, Raible D, Geba GP and Molfino NA: Biology of the interleukin-9 pathway and its therapeutic potential for the treatment of asthma. Inflamm Allergy Drug Targets 10: 180-186, 2011.

10. Kim MS, Cho KA, Cho YJ and Woo SY: Effects of interleukin-9 blockade on chronic airway inflammation in murine asthma models. Allergy Asthma Immunol Res 5: 197-206, 2013.

11. Angkasekwinai $\mathrm{P}$, Srimanote $\mathrm{P}$, Wang $\mathrm{YH}$, et al: Interleukin-25 (IL-25) promotes efficient protective immunity against Trichinella spiralis infection by enhancing the antigen-specific IL-9 response. Infect Immun 81: 3731-3741, 2013.

12. Khan WI, Richard M, Akiho H, et al: Modulation of intestinal muscle contraction by interleukin-9 (IL-9) or IL-9 neutralization: Correlation with worm expulsion in murine nematode infections. Infect Immun 71: 2430-2438, 2003.

13. Li H and Rostami A: IL-9: Basic biology, signaling pathways in $\mathrm{CD}^{+} \mathrm{T}$ cells and implications for autoimmunity. J Neuroimmune Pharmacol 5: 198-209, 2010.

14. Yang Y, Du L, Sun M, Kijlstra A and Yang P: IFN-beta inhibits the increased expression of IL-9 during experimental autoimmune uveoretinitis. PLoS One 7: e48566, 2012.

15. Leng RX, Pan HF, Ye DQ and Xu Y: Potential roles of IL-9 in the pathogenesis of systemic lupus erythematosus. Am J Clin Exp Immunol 1: 28-32, 2012.

16. Li H, Nourbakhsh B, Ciric B, Zhang GX and Rostami A: Neutralization of IL-9 ameliorates experimental autoimmune encephalomyelitis by decreasing the effector $\mathrm{T}$ cell population. J Immunol 185: 4095-4100, 2010.

17. Purwar R, Schlapbach C, Xiao S, et al: Robust tumor immunity to melanoma mediated by interleukin-9-producing $\mathrm{T}$ cells. Nat Med 18: 1248-1253, 2012.

18. Smith SE, Hoelzinger DB, Dominguez AL, Van Snick J and Lustgarten J: Signals through 4-1BB inhibit T regulatory cells by blocking IL-9 production enhancing antitumor responses. Cancer Immunol Immunother 60: 1775-1787, 2011.

19. Sun GG, Wang YD, Cui DW, Cheng YJ and Hu WN: Epithelial membrane protein 1 negatively regulates cell growth and metastasis in colorectal carcinoma. World J Gastroenterol 20: 4001-4010, 2014

20. Cerkovnik P, Perhavec A, Zgajnar J and Novakovic S: Optimization of an RNA isolation procedure from plasma samples. Int J Mol Med 20: 293-300, 2007.

21. Livak KJ and Schmittgen TD: Analysis of relative gene expression data using real-time quantitative PCR and the 2(-Delta Delta C(T)) method. Methods 25: 402-408, 2001.

22. Ouyang H, Shi Y, Liu Z, Feng S, et al: Increased interleukin-9 and CD4 +IL-9+ T cells in patients with systemic lupus erythematosus. Mol Med Rep 7:1031-1037, 2013

23. de Vries VC, Wasiuk A, Bennett KA, et al: Mast cell degranulation breaks peripheral tolerance. Am J Transplant 9: 2270-2280, 2009.

24. Fábrega E, López-Hoyos M, San Segundo D, et al: Interleukin-9 in stable liver transplant recipients. Transplant Proc 44: 1536-1538, 2012.

25. Zhang J, Wang WD, Geng QR, et al: Serum levels of interleukin-9 correlate with negative prognostic factors in extranodal NK/T-cell lymphoma. PLoS One 9: e94637, 2014.

26. Lv X, Feng L, Fang X, Jiang Y and Wang X: Overexpression of IL-9 receptor in diffuse large B-cell lymphoma. Int J Clin Exp Pathol 6: 911-916, 2013.

27. Hamre H, Zeller B, Kanellopoulos A, et al: Serum cytokines and chronic fatigue in adults surviving after childhood leukemia and lymphoma. Brain Behav Immun 30: 80-87, 2013.

28. Kobayashi H, Kumai T, Hayashi S, et al: A naturally processed HLA-DR-bound peptide from the IL-9 receptor alpha of HTLV-1-transformed T cells serves as a T helper epitope. Cancer Immunol Immunother 61: 2215-2225, 2012.

29. Fischer M, Bijman M, Molin D, et al: Increased serum levels of interleukin-9 correlate to negative prognostic factors in Hodgkin's lymphoma. Leukemia 17: 2513-2516, 2003.

30. Hornakova T, Staerk J, Royer Y, et al: Acute lymphoblastic leukemia-associated JAK1 mutants activate the Janus kinase/STAT pathway via interleukin-9 receptor alpha homodimers. J Biol Chem 284: 6773-6781, 2009.

31. Qiu L, Lai R, Lin Q, et al: Autocrine release of interleukin-9 promotes Jak3-dependent survival of $\mathrm{ALK}^{+}$anaplastic large-cell lymphoma cells. Blood 108: 2407-2415, 2006. 
32. Knoops L and Renauld JC: IL-9 and its receptor: from signal transduction to tumorigenesis. Growth Factors 22: 207-215, 2004.

33. Bauer JH, Liu KD, You Y, Lai SY and Goldsmith MA Heteromerization of the gammac chain with the interleukin-9 receptor alpha subunit leads to STAT activation and prevention of apoptosis. J Biol Chem 273: 9255-9260, 1998.

34. Ye ZJ, Zhou Q, Yin W, Yuan ML, et al: Differentiation and immune regulation of IL-9-producing $\mathrm{CD}^{+} \mathrm{T}$ cells in malignant pleural effusion. Am J Respir Crit Care Med 186: 1168-1179, 2012.
35. Lu Y, Hong S, Li H, et al: Th9 cells promote antitumor immune responses in vivo. J Clin Invest 122: 4160-4171, 2012.

36. Tran E, Nielsen JS, Wick DA, et al: Polyfunctional T-cell responses are disrupted by the ovarian cancer ascites environment and only partially restored by clinically relevant cytokines. PLoS One 5: e15625, 2010. 